\title{
Space-Weather capabilities and preliminary results of the High Energy Particle Detector (HEPD) on board the CSES-01 satellite
}

\author{
M. Martucci ${ }^{* a}$, G. Ambrosi ${ }^{c}$, R. Battiston ${ }^{d, e}$, S. Bartocci ${ }^{a, k}$, L. Basara ${ }^{d}$, W.J. Burger ${ }^{d}$, \\ D. Campana $^{f}$, L. Carfora ${ }^{a, b}$, M. Casolino ${ }^{d}$, G. Castellini ${ }^{m}$, P. Cipollone ${ }^{a}$, L. Conti $^{a, k}, \mathbf{A}$. \\ Contin $^{h, i}$, C. De Donato ${ }^{a}$, F. De Persio ${ }^{a}$, C. De Santis ${ }^{a}$, B. Di Ruzza ${ }^{d, e}$, F.M. Follega ${ }^{d, e}$, \\ C. Guandalini ${ }^{h}$, M. Ionica ${ }^{c}$, R. luppa ${ }^{d, e}$, G. Laurenti $^{h}$, I. Lazzizzera ${ }^{d, e}$, M. Lolli ${ }^{h}$, C. \\ Manea $^{d}$, L. Marcelli ${ }^{a}$, G. Masciantonio ${ }^{a}$, M. Mergé ${ }^{a}$, G. Osteria ${ }^{f}$, L. Pacini ${ }^{m}$, F. \\ Palma $^{a, b}$, F. Palmonari ${ }^{h, i}$, B. Panico ${ }^{f}$, A. Parmentier ${ }^{a}$, L. Patrizii ${ }^{h}$, F. Perfetto ${ }^{f}$, P. \\ Picozza $^{a, b}$, M. Piersanti ${ }^{a}$, M. Pozzato ${ }^{h}$, M. Puel ${ }^{d}$, I. Rashevskaya ${ }^{d}$, E. Ricci ${ }^{d, e}$, M.

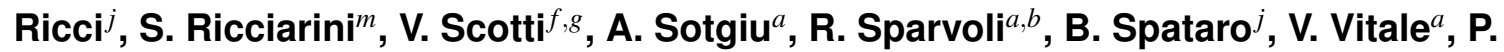 \\ Zuccon $^{d, e}, \mathbf{S}$. Zoffoli ${ }^{m}$ \\ ${ }^{a}$ INFN - Sezione di Roma Tor Vergata, V. della Ricerca Scientifica 1, 00133, Rome, Italy \\ ${ }^{b}$ Università di Roma Tor Vergata, V. della Ricerca Scientifica 1, 00133 Rome, Italy \\ ${ }^{c}$ INFN Sezione di Perugia, V. A. Pascoli, 06123, Perugia, Italy \\ ${ }^{d}$ INFN - TIFPA, V. Sommarive 14, 38123 Povo (Trento), Italy \\ ${ }^{e}$ Università di Trento, V. Sommarive 14, 38123 Povo (TN), Italy \\ ${ }^{f}$ INFN - Sezione di Napoli, V. Cintia, 80126, Naples, Italy \\ ${ }^{g}$ Università di Napoli Federico II, C.so Umberto I, 40, 80138, Naples, Italy \\ ${ }^{h}$ INFN - Sezione di Bologna, V.le Berti Pichat 6/2, Bologna, Italy \\ ${ }^{i}$ Università di Bologna, V.le Berti Pichat 6/2, Bologna, Italy \\ jINFN - LNF, V. E. Fermi, 40, 00044 Frascati (RM), Italy \\ ${ }^{k}$ Uninettuno University, C.so V. Emanuele II, 39, 00186, Rome, Italy \\ ${ }^{l}$ IFAC-CNR, V. Madonna del Piano, 10, 50019 Sesto Fiorentino (FI), Italy \\ ${ }^{m}$ Italian Space Agency, V. del Politecnico, 00133 Rome, Italy \\ E-mail: matteo.martuccieroma2.infn.it
}

(c) Copyright owned by the author(s) under the terms of the Creative Commons

Attribution-NonCommercial-NoDerivatives 4.0 International License (CC BY-NC-ND 4.0). 
CSES-01 (China Seismo-Electromagnetic Satellite) is a mission developed by CNSA (Chinese National Space Administration) and ASI (Italian Space Agency) to investigate the near-Earth electromagnetic, plasma and particle environment, focusing on the disturbances of the ionospheremagnetosphere transition region. In this framework, the Italian High Energy Particle Detector (HEPD) on board CSES-01, is an advanced detector based on a tower of scintillators and a silicon tracker that provide good energy resolution as well as a wide angular acceptance for electrons of 3-100 MeV, protons of 30-200 MeV and light nuclei (up to Oxygen). The very good capabilities in both detection and discrimination of particles make the detector well suited for space-weather purposes; indeed HEPD is able to continuously monitor the magnetospheric environment with high precision and stability in time. Turbulent conditions in the near-Earth environment translate into modification of particles and magnetic/electric measurements at different time-scales. In this work, the data-analysis techniques and some preliminary results concerning the study of different proton populations encountered along CSES-01 orbit (galactic cosmic rays, re-entrant albedo etc.) are presented.

36th International Cosmic Ray Conference -ICRC2019-

July 24th - August 1st, 2019

Madison, WI, U.S.A.

${ }^{*}$ Speaker. 


\section{Introduction}

The terminology Space weather came into popular use in the late 1990s, even though this concept made its debut in the literature many years before. In [1], the expression solar meteorology is used in analogy to terrestrial meteorology, and similar descriptions followed in time [2], [3], [4]. Definitions aside, space weather refers to such effects as reduced satellite operations, failures in spacecraft electronics, radio communication problems from perturbations in the Earth's ionosphere, and even downed power grids during major geomagnetic storms [5]. The whole of these effects can be grouped into two different categories, either directly or indirectly associated with solar activity [7]; in fact, not all space weather effects can be attributed to solar proton events (SPEs). Major solar events (or solar flares) typically generate a wide variety of emissions impacting Earth: X-rays, radio emissions, but also plasma and magnetic field carried by coronal mass ejections or CMEs [6]. Solar energetic particles or SEPs (ions in the $\mathrm{MeV}$ and $\mathrm{GeV}$ energy range and electrons in the $\mathrm{keV}$ and $\mathrm{MeV}$ range) are often produced and accelerated by magnetic reconnection mechanisms [8] or shock phenomena [9].

The impact of both fast and slow CMEs (faster or slower with respect to the heliospheric solar wind) with the Earth magnetosphere led to enhanced electrical currents that cause a subsequent enhancement in the magnetic fields giving birth to geomagnetic storms [6]. The study of such variations is crucial because it would help shed more light on all the mechanisms that take place during solar events and could hopefully led to modelization and, possibly, to create a nowcasting/forecasting network in the nearest future possible. HEPD (High-Energy Particle Detector), on board the CSES-01 satellite, shows outstanding capabilities in monitoring the near-Earth environment, with its high precision and high stability in time.

\section{The instrument}

The High-Energy Particle Detector (HEPD) is one of the nine payloads on board the CSES-01 satellite, developed and maintained by the Italian members of the CSES-Limadou Collaboration. The instrument is optimized for the detection of electrons (3-100 MeV), protons (30-200 MeV), as well as light nuclei, with $\mathrm{Z}$ up to Oxygen's. The payload, with dimensions of approximately $20 \times$ $20 \times 40 \mathrm{~cm}^{3}$, a mass limited to approximately $45 \mathrm{~kg}$ and a power consumption lower than $43 \mathrm{~W}$, comprises four stacked sub-detectors, which are, in descending order:

- the tracker, which is made of two planes of double-side silicon micro-strip sensors providing the direction of the incoming particle;

- the trigger, which is made of one layer of plastic scintillator divided into 6 segments or paddles;

- a calorimeter, including 16 plastic scintillators in the upper section, and 9 LYSO cubic crystals in the lower section. The former section allows to measure both the energy deposition and the range of the impinging particle; the latter provides a larger matter thickness, and it is aimed to increase the operational energy range 

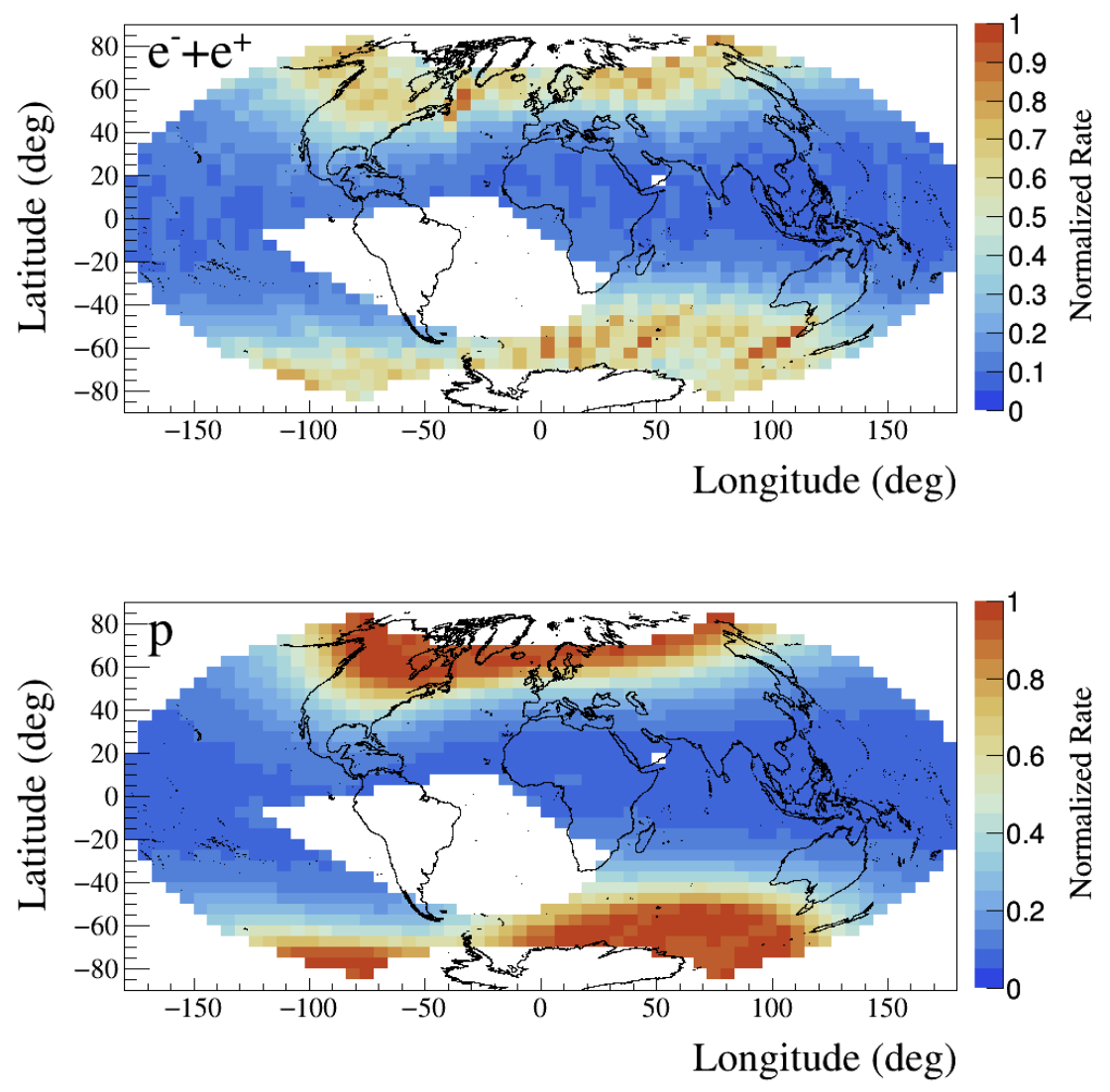

Figure 1: Distribution of electrons/positrons and protons populations as a function of geographical coordinates along CSES-01 orbit (top and bottom panels respectively). South Atlantic Anomaly has been removed from this sample. Data collected by HEPD detector during the period August 20-24, 2018.

- a $5 \mathrm{~mm}$ thick veto system, consisting of five plastic scintill-tor counters (4 of them surrounding the sides of the instrument and one placed in the bottom part). The lateral veto system insures to acquire only particles with trajectories fully contained inside the calorimeter, while the bottom one rejects very energetic particles exiting the instrument or the up-going albedo populations;

The electronics sub-system is inserted in a box placed at one side of the detector and it includes the CPU, the High-Voltage Power Supply and all other mechanical modules employed in the correct functioning of the detector as a whole. For a more detailed description of the instrument, see [10]

\section{The role of HEPD in space weather studies}

The CSES-01 satellite is a 3-axis attitude stabilized spacecraft, moving along a sun-synchronous circular orbit with a $98^{\circ}$ inclination at an altitude of $\sim 500 \mathrm{~km}$. All payload instruments on-board the satellite collect data in the geographic latitude range of $\pm 65^{\circ}$. At higher latitudes all detectors are switched off (adjustment zone), in order to monitor the satellite attitude and manage the orbit 
control system. Though turned off in the adjustment zones, the tilt angle of the Earth magnetic field allows HEPD to collect data in regions with magnetic latitude values of c.a $75^{\circ}$, where the magnetic field lines are almost parallel to each other. Under such circumstances, the geomagnetic rigidity cutoff - which describes the shielding effect of the geomagnetic field, and depends solely on the direction and momentum-to-charge ratio of the incoming particles - is low, and charged particles coming from outside the magnetosphere can penetrate and be easily detected [11].

Using the International Geomagnetic Reference Field (IGRF) series of mathematical models, in particular the IGRF-12 [12], it was possible to obtain the Earth's main field (internal field) at any point of the orbit. In addition, being this field increasingly affected, beyond c.a 4 Earth radii, by the interactions between the magnetosphere and external sources (such as the solar wind), its distortions were taken into account through the implementation of the Tsyganenko- 89 magnetospheric semi-empirical model [13].

The different distributions of protons and electrons/positrons ${ }^{1}$ populations along CSES-01 orbit are shown in the 2 panels of Figure 1 for the period August 20-24, 2018. With an acceptance of $\sim 400 \mathrm{~cm}^{2}$ sr, HEPD can collect a very high statistics even in a short period of time; for example, in a single day of data-taking, HEPD observes almost 500000 electrons/positrons and 3000000 protons. Moreover, since it points along the zenithal direction of pointing, HEPD is vare well suited to measure a wide variety of particles with different origin, such as re-entrant albedo, trapped and cosmic-ray particles. Re-entrant particles are generated with upward-going directions by the interaction of cosmic rays with the Earth's atmosphere, whose trajectories are bent by the geomagnetic field back to the Earth itself [14]. Albedo can be divided in quasi-trapped and un-trapped components: the former is localized in the near-equatorial region [15], inside and below the inner Van Allen belts, the latter spreads over all latitudes [16]. Red-colored regions in the count rate geomaps of Figure 1 correspond to those zones where the rigidity cutoff is low and galactic particles mix together with outer-belt particles. Such outermost regions (which have been highlighted via removal of the South Atlantic Anomaly), contain mostly electrons $(<10 \mathrm{MeV})$ and various types of ions, and are more variable than the inner belt since more easily influenced by solar activity. To study the short-term variability of particles triggered by CMEs impacting the Earth or very strong SEP events (known as Ground Level Enhancements, GLEs, when their energies exceeds $500 \mathrm{MeV}$ ) is important to extract common features. CSES-01 was launched on February 2, 2018 during the minimum of the $24^{\text {th }}$ solar cycle and this should result in a reduction of registered perturbations; nonetheless, powerful SPEs are known to occur in extremely quiet periods as well [17]. Moreover, at the minimum, highly energetic solar particles can travel the Sun-Earth distance with no disturb from a turbulent heliosphere, and this allows for a more precise investigation of their transport mechanisms.

Geomagnetic storms, associated or not with powerful CMEs, can be monitored through different parameters, like the ones shown in Figure 2. One of such proxies is the time profile of the North-South component of the Interplanetary Magnetic Field (IMF), $\mathrm{B}_{z}$; when both $\mathrm{B}_{z}$ and geomagnetic field lines are oriented opposite to each other, they can reconnect, resulting in the transfer of energy from the solar wind to the Earth's magnetosphere. Other proxies include the speed of the

\footnotetext{
${ }^{1}$ Measuring only the energy deposited inside the calorimeter, HEPD is not able to discriminate between electrons and positrons, so the leptonic component is an admixture of the two populations.
} 

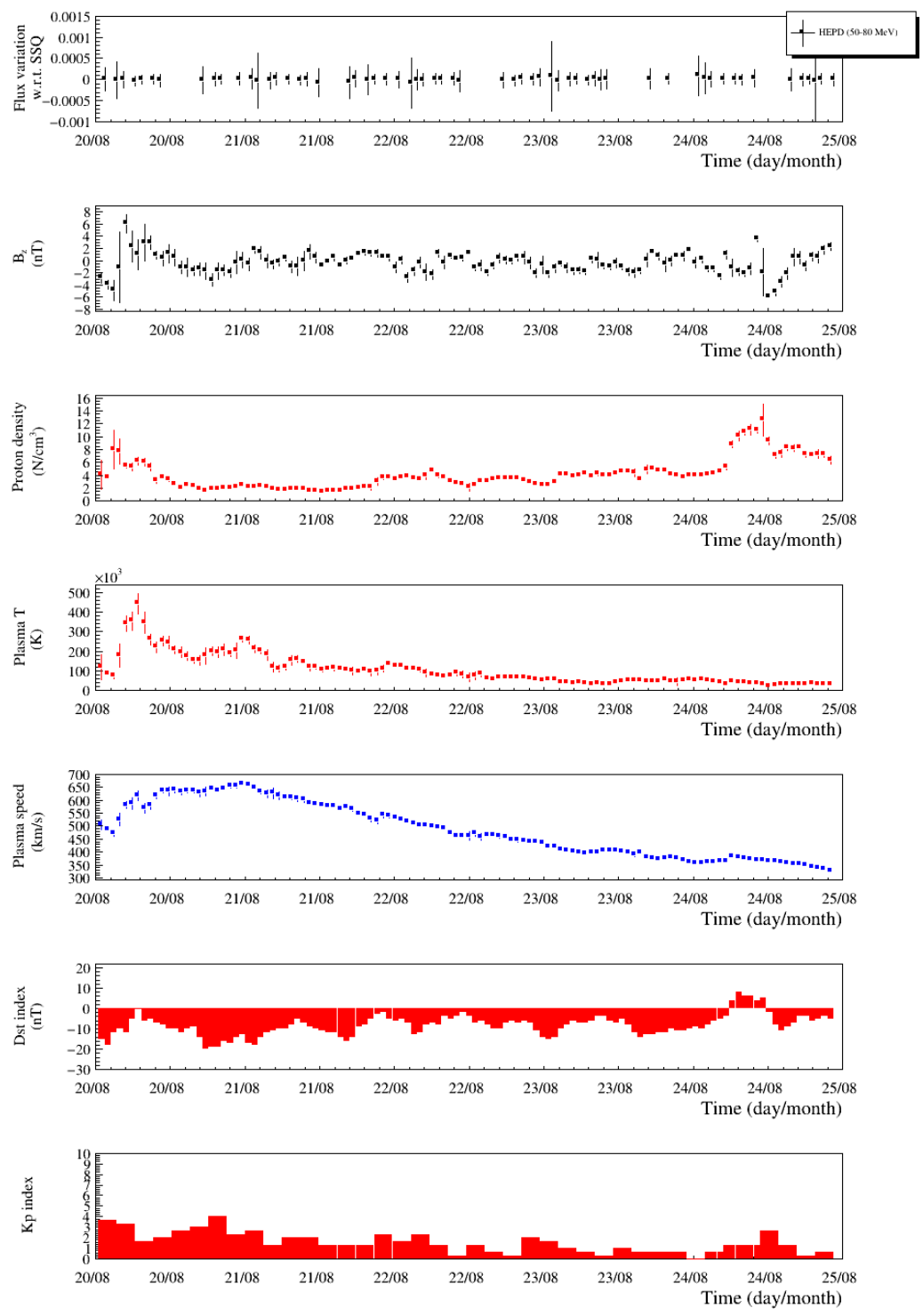

Figure 2: Various useful parameters to monitor possible geomagnetic storms or SPEs from the Sun. From top to bottom: $\mathrm{B}_{z}$ component, proton density, plasma temperature, plasma speed, Dst and $\mathrm{Kp}$ indices, as described in the text (data taken from https://omniweb.gsfc.nasa.gov/). The topmost panel shows the hourly variation of $>50 \mathrm{MeV}$ galactic protons registered by HEPD. HEPD data are normalized with respect to the Super Solar Quiet period (SSQ) of August 9-10, 2018.

solar wind plasma, its temperature and its proton density; under quiet conditions of the interplanetary space, these parameters are often constant or slowly changing, but during the passage of CMEs or other transients from the Sun, they rapidly change in response to the perturbation. Finally, just for comparison, the disturbance storm time index (or Dst) and the $\mathrm{Kp}$ value are shown in the last two panels. The Dst is a hourly parameter that monitors variations of the equatorial magnetospheric ring current ${ }^{2}$, and is derived from the horizontal component of the geomagnetic field as measured

\footnotetext{
${ }^{2}$ It is an electric current carried by charged particles trapped in a planet's magnetosphere.
} 
at ground observatories distant from both the auroral and equatorial ionospheric electrojets, while $\mathrm{Kp}$ is a global 3-hour index, still monitoring the horizontal magnetic field component and derived from ground-based magnetometers at a dozen sub-auroral stations all around the world. All these parameters (obtained from https://omniweb.gsfc.nasa.gov/) are shown in Figure 2, together with the variation of HEPD $>50 \mathrm{MeV}$ galactic protons in the period August 20-24 with respect to the Super Solar Quiet (SSQ) period August 9-10. Some results regarding the G3-level geostorm (associated with a filament eruption) of 2018 August 26 will be presented during the conference.

\section{Conclusions}

The high stability in time, together with the high statistics, make HEPD very well suited for space weather studies. HEPD is able to directly track variations of the cosmic proton population caused by solar injections during SPEs, and also to study the indirect effects of such events on the secondary populations (such as re-entrant albedo). The low energy threshold in electron measurements allows the detection of solar electrons emitted during solar flares [18] and the capabilities in detecting light nuclei could prove very important in the study of phenomena such as the helium enrichment that has been once again observed in various solar flares [19].

\section{References}

[1] Sir J. F. W. Herschel, Results of astronomical observations made during the years 1834, 5, 6, 7, 8, at the Cape of Good Hope; being the completion of a telescopic survey of the whole surface of the visible heavens, commenced in 1825 Smith, Elder and co., London 1847

[2] C. Mackay, The spots on the Sun, London Rev., 1 (1860) 107

[3] E. L. Youmans, The spots on the Sun, Pop. Sci. Mon., 1 (1872) 144

[4] P. Tacchini, Meteorologia Solare, Tipografia Metastasio, Rome 1884

[5] W. B. Cade III et al., The Origin of Space Weather, Space Weather, 13 (2015) 99-103

[6] M. Piersanti et al., Comprehensive Analysis of the Geoeffective Solar Event of 21 June 2015: Effects on the Magnetosphere, Plasmasphere, and Ionosphere Systems, Sol. Phys., 292 (2017) 169

[7] M.A. Shea and D. E. Smart, Space weather, GLEs, Ground-level cosmic ray events, Solar protons, Space Science Review, 171 (2012) 161-188

[8] C. Zhu et al., Observation of the Evolution of a current sheet in a solar flare, ApJ, 821 (2016) L29

[9] V. Petrosian, Particle Acceleration in solar flares and associated CME shocks, ApJ, 830 (2016) 28

[10] P. Picozza et al., Scientic goals and in-orbit performance of the High-Energy Particle Detector on board the CSES satellite, ApJS - in press

[11] G. Lemaitre and M. S. Vallarta, On Compton's Latitude Effect of Cosmic Radiation, wmphPhys. Rev. Lett., 43 (1933) 87

[12] E. Thébault et al., International Geomagnetic Reference Field: the 12th generation, Earth, Planets and Space 67 (2015) 79

[13] N. A. Tsyganenko, A magnetospheric magnetic field model with a wrapped tail current sheet, Planet. Space Sci., 37 (1989) 5-20 
[14] O. Adriani et al., Reentrant albedo proton fluxes measured by the PAMELA experiment, JGR, 120 (2015) 3728-3738

[15] J. Moritz, Energetic protons at low equatorial altitudes, Z. Geophys., 38 (1972) 701-712

[16] J. Alcaraz et al., Protons in near earth orbit, Phys. Lett. B, 472 (2000) 215-226

[17] O. Adriani et al., Observations of the 2006 December 13 and 14 Solar Particle Events in the $80 \mathrm{MeV}$ $n^{-1}-3 \mathrm{GeV} n^{-1}$ Range from Space with the PAMELA Detector, ApJ 742 (2011) 102

[18] G. D. Holman et al., Implications of X-ray observations for electron acceleration and propagation in solar flares, Space Sci. Rev., 159 (2011) 107-166

[19] J. Hirshberg et al. Solar flares and solar wind helium enrichments: July 1965 July 1967, Solar Physics, 23 (1972) 467-486 